\title{
BANACH ALGEBRAS WHICH ARE NOT WEDDERBURNIAN
}

\author{
BERTRAM YOOD
}

(Communicated by Palle E. T. Jorgensen)

\begin{abstract}
Let $A$ be a Banach algebra with radical $R$. In 1951 Feldman exhibited an example in which it is impossible to find a closed subalgebra $K$ of $A$ such that $A=K \oplus R$. We provide other examples. Feldman's algebra is commutative, but these examples are, in general, not commutative.
\end{abstract}

\section{INTRODUCTION}

In [5, p. 85] Glaeser called a Banach algebra $A$ Wedderburnian if $A$ is the direct sum of its radical $R$ and a closed subalgebra $K$ of $A$. In [2] Bade and Curtis called such a Banach algebra strongly decomposable. If $A$ is finite dimensional then a classical result of Wedderburn shows that $A$ is Wedderburnian. In [4] Feldman provided an example where $A$ is not Wedderburnian. This algebra was studied in detail in [1]. For another example see [5]. These examples are commutative. Our aim is to provide noncommutative examples which occur rather naturally. Some instances arise as follows. Let $G$ be an infinite compact topological group with identity $e$. Let $C(G)$ be the set of all complexvalued continuous functions on $G$ taken as an algebra with convolution as its multiplication. Let $\|f\|_{2}$ be the $L^{2}$-norm of $f \in C(G)$. The norm

$$
|| f \mid \|=\max \left(\|f\|_{2},|f(e)|\right)
$$

is a normed algebra norm on $C(G)$. The completion $A$ of $C(G)$ in this norm is not Wedderburnian. The Feldman example can be identified with the completion of the socle of $C(G)$, for $G$ the reals modulo one, in the norm $|\|f \mid\|$.

Other examples arising from algebras of operators on Hilbert space are given. In particular, the completion of the trace class [9, p. 37] of Schatten in an appropriate norm is not Wedderburnian.

\section{Preliminary theory}

We adopt the following notation. Let $B$ be a Banach algebra in the norm $\|x\|$ and $E$ be a Banach space in the norm $\|\xi\|_{E}$. Let $T$ be a linear mapping of $B$ into $E$ satisfying

$$
\|T(x y)\|_{E} \leq\|x\|\|y\|
$$

Received by the editors December 2, 1991.

1991 Mathematics Subject Classification. Primary 46H10. 
for all $x, y$ in $B$. We let $A$ be the set of all elements of the form $x+\xi$, where $x \in B$ and $\xi \in E$, made into an algebra under the rules $(x+\xi)+(y+\eta)=$ $(x+y)+(\xi+\eta), a(x+\xi)=(a x+a \xi)$, and $(x+\xi)(y+\eta)=x y$ for all $x, y \in B$, $\xi, \eta \in E$, and scalars $a$.

We define a norm on $A$ by

$$
\|\| x+\xi \mid \|=\max \left(\|x\|,\|\xi-T(x)\|_{E}\right) .
$$

In view of (1) we see that $\|x+\xi\| \|$ is a normed algebra norm on $A$.

2.1. Lemma. $A$ is a Banach algebra in the norm $\|x+\xi\| \|$.

Proof. Let $\left\{x_{n}+\xi_{n}\right\}$ be a Cauchy sequence in $A$. Then $\left\{x_{n}\right\}$ is a Cauchy sequence in $B$ and $\left\{T\left(x_{n}\right)-\xi_{n}\right\}$ is a Cauchy sequence in $E$. Hence there exists $y \in B$ and $\eta \in E$ where $\left\|x_{n}-y\right\| \rightarrow 0$ and $\left\|T\left(x_{n}\right)-\xi_{n}-\eta\right\|_{E} \rightarrow 0$. One readily checks that, in $A$, the sequence $x_{n}+\xi_{n}$ has $y+[T(y)-\eta]$ as its limit.

We denote the radical of $A$ by $R$.

2.2. Lemma. If $B$ is semisimple then $R=E$.

Proof. Clearly $E \subset R$. Note that $B$ is a two-sided ideal in $A$. Therefore, $R \cap B$ is the radical of $B$ so that $R \cap B=(0)$. Let $x+\xi \in R$ where $x \in B$ and $\xi \in E$. Then $x \in R \cap B$ so that $x=0$.

2.3. Lemma. Suppose that $T$ is discontinuous on a linear subspace $W$ of $B$. If $E$ is finite dimensional then the closure of $W$ in $A$ must contain a nonzero element of $E$.

Proof. There exists a sequence $\left\{x_{n}\right\}$ in $W$ where $\left\|x_{n}\right\| \rightarrow 0$ and $\left\|T\left(x_{n}\right)\right\|_{E}=1$ for each $n=1,2, \ldots$. As $E$ is finite dimensional, there is a subsequence $\left\{y_{n}\right\}$ of $\left\{x_{n}\right\}$ and some $\xi \neq 0$ in $E$ such that $\left\|\xi-T\left(y_{n}\right)\right\|_{E} \rightarrow 0$. Then, since

$$
\left\|\mid y_{n}+\xi\right\| \|=\max \left(\left\|y_{n}\right\|,\left\|\xi-T\left(y_{n}\right)\right\|_{E}\right),
$$

we see that $-\xi$ is in the closure of $W$ in $A$.

2.4. Theorem. Suppose that $B$ is semisimple and that $E$ is finite dimensional. Suppose that $W$ is a two-sided ideal in $B$ and that $T$ is discontinuous on $W^{2}$. Then the completion $V$ of $W$ in the norm

$$
\||| x \mid\|=\max \left(\|x\|,\|T(x)\|_{E}\right)
$$

is a Banach algebra which is not Wedderburnian.

Proof. By Lemma 2.1, $V$ is just the closure of $W$ in the Banach algebra $A$. Also $V$ is a two-sided ideal in $A$ so that, by Lemma 2.2, the radical $S$ of $V$ is $V \cap E$.

Suppose that $V=K \oplus S$ where $K$ is a subalgebra of $V$. Since $s v=v s=0$ for all $s \in S$ and $v \in V$, we have $K \supset V^{2} \supset W^{2}$. Hence, by Lemma 2.3, the closure of $K$ in $V$ must contain a nonzero element of $S$. Consequently $V$ is not Wedderburnian.

\section{EXAMPLES FROM HARMONIC ANALYSIS}

Let $G$ be an infinite compact topological group with identity $e$ and normalized Haar measure $m(E)$. We consider $C(G)$ in the sup norm and $L^{2}(G)$ in 
the $L^{2}$-norm $\|f\|_{2}$ as Banach algebras with convolution $f * g$ as the multiplication. If $f$ and $g$ are in $L^{2}(G)$ then $f * g$ lies in $C(G)$ by [6, p. 295]. Therefore, the socle $\mathfrak{S}$ of $L^{2}(G)$ lies in $C(G)$, and $\mathfrak{S}$ is also the socle of $C(G)$. As $C(G)$ is a dual algebra [7, Theorem 15], $\mathfrak{S}$ is dense in $C(G)$ as well as $L^{2}(G)$.

We use the standard description of $\mathfrak{S}$ provided by the Peter-Weyl theorem. Let $\Lambda$ be the set of equivalence classes of finite-dimensional irreducible representations of $G$. For each $\alpha \in \Lambda$ we select an irreducible unitary representation $R^{\alpha}$ in the class $\alpha$. Suppose $R^{\alpha}(t)$ is the $n_{\alpha}$ by $n_{\alpha}$ matrix $\left(D_{i j}^{\alpha}(t)\right)$. Then $\mathfrak{S}$ consists of all linear combinations of the functions $D_{i j}^{\alpha}, \alpha \in \Lambda$, $i, j=1, \ldots, n_{\alpha}$. The functions $n_{\alpha}^{1 / 2} D_{i j}^{\alpha}$ form an orthonormal basis for $L^{2}(G)$. Also $D_{i j}^{\alpha} * D_{r s}^{\beta}=0$ if $\alpha \neq \beta$ and

$$
n_{\alpha} D_{i j}^{\alpha} * n_{\alpha} D_{r s}^{\alpha}=n_{\alpha} \delta_{j r} D_{i s}^{\alpha},
$$

where $\delta_{j r}$ is the Kronecker delta.

Let $\mathfrak{D}$ be the set of all linear combinations of the "diagonal" entries $D_{i i}^{\alpha}$, $\alpha \in \Lambda, i=1, \ldots, n_{\alpha}$. The convolution of two different diagonal entries is zero and each $D_{i i}^{\alpha}$ is a scalar multiple of an idempotent. Therefore, $\mathfrak{D}$ is a commutative subalgebra of $C(G)$.

3.1. Lemma. Consider $\mathfrak{D}$ as a normed algebra in the $L^{2}$-norm $\|f\|_{2}$. Then the linear functional $f \rightarrow f(e)$ is discontinuous on $\mathfrak{D}$.

Proof. Let

$$
f=\sum_{k=1}^{r} k^{-1} D_{i_{k} i_{k}}^{\alpha_{k}}
$$

be in $\mathfrak{D}$ where the $D_{i_{k} i_{k}}^{\alpha_{k}}$ are different diagonal entries. Then $f(e)=\sum_{k=1}^{r} k^{-1}$ and $\|f\|_{2}=\sum_{k=1}^{r} k^{-2} n_{\alpha_{k}}^{-1 / 2}$.

3.2. Lemma. The closure of $\mathfrak{D}$ in either $C(G)$ or $L^{2}(G)$ is semisimple.

Proof. Note that $v=n_{\alpha}^{1 / 2} D_{i i}^{\alpha}$ is an idempotent generator of a minimal onesided ideal of $C(G)$ or $L^{2}(G)$. Let $W$ be the closure of $\mathfrak{D}$ and $z$ be in the radical of $W$. We have, as $W$ is commutative, that $v z=z v=v z v$ is a scalar multiple of the idempotent $v$ and is in the radical of $W$. Hence $D_{i i}^{\alpha} * z=0$. It follows from (2) that $D_{r s}^{\alpha} * z=0$ for all $\alpha \in \Lambda$ and $r, s=1, \ldots, n_{\alpha}$. Hence $\mathfrak{S} * z=z * \mathfrak{S}=(0)$. Since $\mathfrak{S}$ is dense in $C(G)$ and $L^{2}(G)$ and these are semisimple, we see that $z=0$.

3.3. Notation. The functional $f \rightarrow f(e)$ which is defined naturally on $C(G)$ can be extended to a linear functional $\phi(f)$ defined on $L^{2}(G)$ by [10, Theorem 1.71-A, p. 40].

3.4. Lemma. For any $f, g \in L^{2}(G)$ we have

$$
|\phi(f * g)| \leq\|f\|_{2}\|g\|_{2} .
$$

Proof. As noted earlier, $f * g \in C(G)$. Therefore,

$$
|\phi(f * g)|=|f * g(e)| \leq\|f\|_{2}\|g\|_{2}
$$

by Schwarz's inequality. 
3.5. Theorem. Let $K$ be either the closure of $\mathfrak{D}$ in $L^{2}(G)$ or any two-sided ideal of $L^{2}(G)$ containing $\mathfrak{D}$. Then the completion of $K$ in the norm

$$
\|\| f \mid \|=\max \left[\|f\|_{2},|\phi(f)|\right]
$$

is a Banach algebra which is not Wedderburnian.

Proof. Clearly $\mathfrak{D}^{2}=\mathfrak{D}$. Theorem 3.5 follows from Lemmas $3.1,3.2$, and 3.4 together with Theorem 2.4. The special case $K=C(G)$ was mentioned in $\S 1$.

For the case $K=\mathfrak{S}$ we have a specific result.

3.6. Corollary. Let $f$ be a typical element of $\mathfrak{S}$ where

$$
f=\sum_{k=1}^{r} a_{k} D_{i_{k} j_{k}}^{\alpha_{k}}
$$

Here each $a_{k}$ is a scalar and no two $D_{i j}^{\alpha}$ agree in all of $\alpha, i$, and $j$. Then the completion of $\mathfrak{S}$ in the norm

$$
\||f|\|=\max \left\{\left(\sum_{k=1}^{r}\left|a_{k}\right|^{2} / n_{\alpha_{k}}\right)^{1 / 2},\left|\sum_{k=1}^{r} a_{k} \delta_{i_{k} j_{k}}\right|\right\}
$$

is not Wedderburnian.

Proof. We use Theorem 3.5 together with $D_{i j}^{\alpha}(e)=\delta_{i j}$.

If $G$ is abelian each $m_{\alpha}=1$ and each $\delta_{i j}=1$. Here $\mathfrak{S}$ is the set of linear combinations of the continuous characters of $G$.

3.7. Corollary. Let $G$ be an abelian compact group whose character group $\widehat{G}$ is denumerably infinite: $\widehat{G}=\left\{\gamma_{1}, \gamma_{2}, \ldots\right\}$. Then the completion of $\mathfrak{S}$ in the norm

$$
\left\|\left|\sum_{k=1}^{r} a_{k} \gamma_{k} \|\right|=\max \left\{\left(\sum_{k=1}^{r}\left|a_{k}\right|^{2}\right)^{1 / 2},\left|\sum_{k=1}^{r} a_{k}\right|\right\}\right.
$$

is not Wedderburnian.

For $G$ the reals modulo one we have, except for a difference in notation, the Feldman example [4].

\section{EXAMPLES FROM OPERATOR THEORY}

Let $B(H)$ be the algebra of all bounded linear operators on a separable infinite-dimensional Hilbert space $H$. Let $\left\{\phi_{n}\right\}$ be an orthonormal basis for $H$. As in Schatten's book [9] (see also [3, Chapter 1]) we consider the Schmidt class $B_{2}$ and the trace-class $B_{1}$ of operators on $H . B_{2}$ is the set of all $T \in$ $B(H)$ for which $\sum_{j}\left\|T\left(\phi_{j}\right)\right\|^{2}<\infty$. This sum is finite and the same if $\left\{\phi_{n}\right\}$ is replaced by another orthononormal basis $\left\{\psi_{n}\right\}$. As shown in [9], $B_{2}$ is a Banach *-algebra in the norm

$$
\|T\|_{2}=\left[\sum_{j}\left\|T\left(\phi_{j}\right)\right\|^{2}\right]^{1 / 2} .
$$

Also $\|T\|_{2}=\left\|T^{*}\right\|_{2}$ for all $T \in B_{2}$. 
Let $|T|$ be the unique positive square root of $T^{*} T$. The trace-class $B_{1}$ is the set of all $T \in B(H)$ for which $\sum_{j}\left(|T|\left(\phi_{j}\right), \phi_{j}\right)<\infty$. Again this sum is finite and the same if $\left\{\phi_{n}\right\}$ is replaced by another orthonormal basis $\left\{\psi_{n}\right\}$. As shown in [9], $B_{1}$ is a Banach $*$-algebra under the norm

$$
\|T\|_{1}=\sum_{j}\left(|T|\left(\phi_{j}\right), \phi_{j}\right) .
$$

Furthermore, $B_{1}$ is the set of all products $T U$ for $T, U \in B_{2}$, and the elements of $B_{1}$ all have a finite trace

$$
\operatorname{tr}(U)=\sum_{j}\left(U\left(\phi_{j}\right), \phi_{j}\right), \quad U \in B_{1},
$$

again independent of the choice of the orthonormal basis. By [10, 1.71-A, p. 40], $\operatorname{tr}(U)$ can be extended to be a linear functional $T R(U)$ on all of $B_{2}$.

We note that (see [9]) the common socle of $B_{1}$ and $B_{2}$ is the set $F(H)$ of all $U \in B(H)$ with finite-dimensional range.

4.1. Lemma. For $U, V \in B_{2}$ we have

$$
|T R(U V)| \leq\|U\|_{2}\|V\|_{2} .
$$

Proof. As noted above, $U V \in B_{1}$. Therefore,

$$
\begin{aligned}
|T R(U V)| & =\left|\sum_{j}\left(U V\left(\phi_{j}\right), \phi_{j}\right)\right| \leq \sum_{j}\left|\left(V\left(\phi_{j}\right), U^{*}\left(\phi_{j}\right)\right)\right| \\
& \leq \sum_{j}\left\|V\left(\phi_{j}\right)\right\|\left\|U^{*}\left(\phi_{j}\right)\right\| \leq\|V\|_{2}\left\|U^{*}\right\|_{2}=\|V\|_{2}\|U\|_{2} .
\end{aligned}
$$

4.2. Lemma. $\operatorname{tr}(U)$ is discontinuous on $F(H)$ if $F(H)$ is taken in the norm $\|U\|_{2}$.

Proof. For each positive integer $n$ we define $W_{n} \in F(H)$ as follows. Let $W_{n}\left(\phi_{j}\right)=\phi_{j} / j$ for $j=1, \ldots, n$ and $W_{n}\left(\phi_{j}\right)=0$ for $j>n$. Then

$$
\operatorname{tr}\left(W_{n}\right)=\sum_{j=1}^{n} j^{-1} \text { and }\left\|W_{n}\right\|_{2}=\sum_{j=1}^{n} j^{-2} .
$$

4.3. Theorem. Let $K$ be any two-sided ideal of $B_{2}$ which contains $F(H)$. The completion of $K$ in the norm

$$
|||V| \|=\max \left(\|V\|_{2},|T R(V)|\right)
$$

is not Wedderburnian.

Proof. Note that $F(H)=[F(H)]^{2}$. We can then use Lemmas 4.1 and 4.2 to apply Theorem 2.4 . The particular case $K=B_{1}$ was noted in $\S 1$.

Consider the specific case $H=l_{2}$. Any $V \in B\left(l_{2}\right)$ can be described in matrix terms. There corresponds to $V$ an infinite matrix $\left[v_{r s}\right]$ so that, for $x=\left\{x_{n}\right\}$ and $y=\left\{y_{n}\right\}$ in $l_{2}, V(x)=y$ if and only if

$$
y_{r}=\sum_{s=1}^{\infty} v_{r s} x_{s}, \quad r=1,2, \ldots
$$


For $V$ we have

$$
\|V\|_{2}=\left[\sum_{j} \sum_{j}\left|v_{i j}\right|^{2}\right]^{1 / 2}, \quad \operatorname{tr}(V)=\sum_{j} v_{j j}
$$

In these terms $B_{2}$ is the set of all $V \in B\left(l_{2}\right)$ for which $\sum_{j} \sum_{i}\left|v_{i j}\right|^{2}<\infty$, and although there seems to be no simple description of $B_{1}$ in matrix terms (see [8, p. 107]), $F\left(l_{2}\right)$ is easily described as all $V \in B_{2}$ for which the column vectors of the matrix $\left[v_{r s}\right]$ lie in a finite-dimensional subspace of $l_{2}$.

4.4. Corollary. The completion of $F\left(l_{2}\right)$ in the normed algebra norm

$$
\|V \mid\|=\max \left\{\left[\sum_{j} \sum_{i}\left|v_{i j}\right|^{2}\right]^{1 / 2},\left|\sum_{j} v_{j j}\right|\right\}
$$

is not Wedderburnian.

\section{ADDED IN PROOF}

Interesting examples of commutative Banach algebras not Wedderburnian were given by G. F. Bachelis and S. Saeki, Banach algebras with uncomplemented radical, Proc. Amer. Math. Soc. 100 (1987), 271-273.

\section{REFERENCES}

1. W. G. Bade and P. C. Curtis, Homomorphisms of commutative Banach algebras, Amer. J. Math. 82 (1960), 589-608.

2. _ The Wedderburn decomposition of commutative Banach algebras, Amer. J. Math. 82 (1960), 851-861.

3. J. B. Conway, The theory of subnormal operators, Math. Surveys Monographs, vol. 36, Amer. Math. Soc., Providence, RI, 1991.

4. C. Feldman, The Wedderburn principal theorem in Banach algebras, Proc. Amer. Math. Soc. 2 (1951), 771-777.

5. G. Glaeser, Étude de certaines algèbres Taylorienne, J. Analyse Math. 6 (1958), 1-124.

6. E. Hewitt and K. A. Ross, Abstract harmonic analysis, vol. I, Springer-Verlag, New York, 1963.

7. I. Kaplansky, Dual rings, Ann. of Math. (2) 49 (1948), 689-701.

8. J. R. Ringrose, Compact non-self-adjoint operators, Van Nostrand Reinhold, New York, 1971.

9. R. Schatten, Norm ideals of complete by continuous operators, Springer-Verlag, Berlin, 1960.

10. A. E. Taylor, Introduction to functional analysis, Wiley, New York, 1958.

Department of Mathematics, Pennsylvania State University, University Park, PennSYLVANIA 16802 